\title{
Sense Analysis and Application in Fashion Models Performance
}

\author{
LIU Wenbo ${ }^{1}$, SHI Jianping ${ }^{2 *}$, Ting-Ting Wang ${ }^{2}$ \\ ${ }^{1}$ College of Clothing and Textile, Liaodong College, Dandong 118003, China \\ ${ }^{2}$ College of Clothing and Textile Engineering, Soochow University, Suzhou 215021, China
}

\begin{abstract}
This paper, from the perspective of the sense of fashion models, analyzes inner sense which contains kinesthetic and balance sense; external sense; sense of personality and contrast. It interprets the application of fashion model performance from four aspects: fashion dressing, selfexpression, body show, and space environment.the value of fashion model's performing sense is a kind of extraordinary play by studies. This exceptional performance is actually the result of model's stage experiences accumulated year by year,which provides the sense of culture connotation and unique fascination to the modern fashion show.
\end{abstract}

Keywords:Fashion model; Performance; Sense analysis; Application

\section{INTRODUCTION}

Fashion model relies on shape of the body, image and skills to present the characteristics of fashion styles and space environment. Fashion models often pay high attention to the "kinesthetic sense", "performing sense"and"sense of fashion". And in a show, the audiences will normally complement those models who deliver "good sense". Then, what does "sense" exactly mean?

In Chinese dictionary,"sense"is described as a physiological capacity of brains that provides data for perception, especially when sensory organs are excited by objects.
In general psychology, "sense"is considered to be a simple psychological phenomenon. That is, the direct effect of objects to human sensory organs, and thus human brains react to these individual properties about the objects. This reaction is referred to as sense [1]. The sense of fashion model is composed of variety of factors, which include inner sense, external sense, personality sense and contrast sense.Sense is vital to fashion models performance. It's an effective way to enhance models' literacy.

\section{Analysis of fashion model's sense}

\subsection{Inner sense---kinesthetic sense and vestibular sense}

Inner sense also known as organic sense, refers to the feeling that reflects the internal state and changes of the organs. Inner sense can be split into two aspects: kinesthetic sense and vestibular sense.

(1) Kinesthetic sense is not only the feelings generated from physical activity, but also information feedback obtained from the process of recognition. This information feedback provides kinesthetic awareness to fashion models during their physical movements. Kinesthetic sense allows fashion models to better perceive the location, postures and position of each part of their body. Figure 1 shows that the fashion model uses postures, walking or even a wise turn-back in their presentation to attract audiences, bring a chord with the 
audiences and end up with a contagion effect that transmitted the keen enjoyment of beauty to the audiences.

(2) Vestibular sense,also known as static sense refers to the stationary state of the modeling when fashion models maintain a stationary stand of their body during the fashion show. Shown in figure 2, regardless how the model moves, the center of gravity of the body is always in equilibrium. This gives a feeling of pleasant.

During the fashion show,kinesthetic sense and vestibular sense have always been the determinants of the dynamic state and the static state of fashion models. It is essential for fashion models to grasp interdependency and mutual constraint between kinesthetic sense and vestibular sense, strive to bring out the flawless feelings that combine both dynamic and static sense.

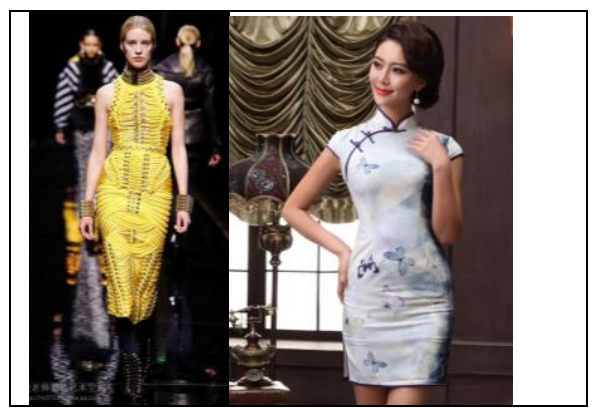

Fig.1 State of Kinesthetic sense, liveliness and vividness

Fig.2 Static state of balance, full of perception

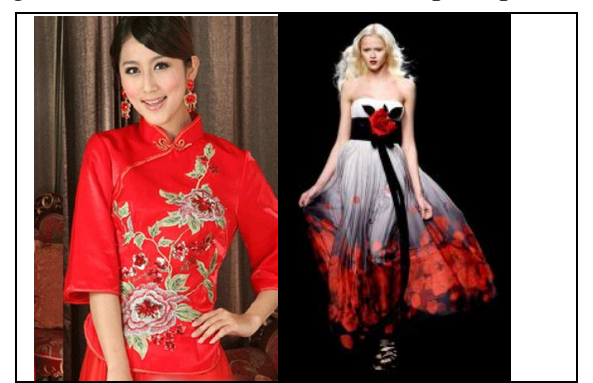

Fig.3 Comparison of red and green, a foil for peony pattern
Fig.4 Noble manner, dignified sight

\subsection{External sense}

External sense usually refers to the integrated feeling that the fashion model generated from the stage environment, coworkers and the audiences. This integrated feeling includes hearing, sight and tactile etc. When fashion models receive the message from the surrounding environment, the stage atmosphere and musical sound, they conduct physical movements such as the changes on their body, mood and emotions through external sense, in order to achieve the resonance effect with feelings.

The external sense of fashion models is based on their performance awareness, body language and the ability to understand and perceive the music. Every performance on the stage is to chase and adjust the past experiences, to exploit the potentialities on fashion connotation, to interpret the design concept [2]. External sense of model is actually the meaning of out performance of their past experiences. For instance, why some good looking models with nice figure are not charming on the stage? This relates to the models' past experiences and the ability of understanding. It is also a matter of how they interpret the mood, music, fashion style and the environment. In order to obtain the external sense of performance, models should adjust their mood, understand the music, fashion style and the environment properly, fully control the sensory information to grasp the performing atmosphere and win the glory on the stage.

\subsection{Personality}

Personality is crucial for fashion models. It is not only the reflection of a model's aesthetic ability, but also a model's temperament. Some models do not bound by standard performance. Some ordinary fashion on the contrary can provide more opportunities for the models to express 
their individual feeling and inner cultivation while weakening the defect of the dress at the same time. For instance, different model wearing the same T-shirt will deliver different impression to the audience because of their different personalities, color, stature and appearance. Some are noble, dignified and free and easy. Some are rough and wild, leading the edge and lively. It's crucial for the fashion models to wear something that fits them. It will make it easier to express their personality. And it can not only meet the character and style of the fashion, but also reflect the model's inner world. However, it would be unacceptable if models only stick into their own personality without considering the original intention of the fashion design, or even overriding the fashion. Therefore, fashion models should weigh the relationship between "personality and fashion", to reach the perfect unity between personal feeling and purpose of the fashion design on the $\mathrm{T}$ stage.

\subsection{Contrast sense}

Contrast sense refers to the feeling of more than two styles of fashion which are combined together in the performance of fashion show. For example, under the theme of "fewer stars and the bright moonlight", the star patterns on the cloth are relatively fewer under the bright moonlight. And they become more and more while in the darkness. This is the contrast sense. Again, let's do a color test. Pick up two green Qipao with same size and style, then leave them on the blue and yellow background respectively. The one on the blue background looks slightly yellower, and the one on the yellow background looks bluish. We find the contrast is especially obvious in the vicinity of two-color intersection, provide a feeling of visual stimulus to people.
It is proved that there is contrast sense in color. This principle of contrast is often used in fashion show. For example, the backgrounds are often dark when clothes are light in the fashion show and vice versa. If designer wants audience to pick up the key points on the costume, then the contrast colors of green and red, blue and yellow can be used to stimulate the visual impact. As shown in figure 3 , in the front collar part, designer uses green leaves set off two peony pattern in red and green, two peonies stand out under the contrast, bring out a feeling of elegant and graceful.

\section{The sense application in fashion show}

\subsection{Self-expression}

Self-expression in psychology mainly refers to the cognition of individual existence. This cognition is a kind of selfexpression that generated from model's psychological activities, psychological status and personality etc. Only when the models have got favorable self-conscious, can they express the "spirit" and "entity" of the fashion that arouse audience's feeling. Favorable self-expression is not a kind of posture, neither mimic, but highly literate. It's also a comprehensive reflection of excellent psychological quality and superb acting skills. As shown in Figure 4, the elegant posture, dignified eyes, it seems not just simply walking, but walking in the heart. The whole body and soul are integrated into the fashion role. The application of this kind of self-expression is able to create elegant demeanor, harmonious and graceful movements, and unrestrained expression. This is the highest state of self-expression.

\subsection{Body dynamic display}

Dynamic display is the most basic form of movements in a performance. Dynamic display can only be brought out by reasonable matching of limbs, torso, head 
and facial features. Body movements that human eyes received can stimulate human retina. It will then act on the brain through the conduction of central nervous system, to arouse the excitement of the cortex and make corresponding reflection [3]. This reaction of human is able to show the dynamic performances temperament, and stimulate the audience which produces feeling of effects. For example, when models are presenting the theme of "pure" in a show, an adolescent girl will come immediately to people's mind. Therefore, as long as the models transform their state to that age, with the feelings flow out naturally without any exaggeration [4], then the feelings of "pure"girl will be presented naturally in the dynamic display. In addition, different emotions such as happiness, excitement, calm, pain and grief can also be expressed through gesture and tread. For example when performing the theme of "festivals", models should move with high spirits, charming smiles and full of happiness. Their movement of arm, waist and leg can be combined with dance move and rate of movement should be increased as well. The happiness, noble and vitality of the lady will be fully interpreted throughout the body movement.

\subsection{Creation of space environment}

The performance venue in the fashion show is one of the conditions that can be used to create a fashionable feeling of the environment, which constitutes the distance between models and audiences. Fashion space environment can be reformed at any time according to the objective of the performance. The design of the space environment should be flexible and appealing, such as the change of the height, morphological changes and division of space entities, so as to create a good visual effect and more comfortable performance environment[5]. By the way of lights, stage technology, photography and even performing routes, the change of spatial pattern, the application of space environment can make people feel the flow of the environment while enjoying the fashion show. For instance, when models are walking on the $U$ shape runway, there is an extended feeling of the space due to the multiple visual angles of the audience. If models are making short stop and turn around on the stage, a strong flow of space environment can be created, which conveys in-depth meaning of the fashion theme and brings a visual sense of beauty in art to the audience.

\section{Conclusion}

Fashion show is a flowing comprehensive discipline. It is an industry derived from fashion culture, as well as Arts that can be appreciated independently. The analysis of the "sense" of fashion models reveals the expression of their inner sense, out sense, personality and contrast sense. And it embodies the soul and essence of the art of fashion show. The ingenious application of fashion sense in the show achieved the application value and performing charm of the four aspects: fashion dress, selfexpression, dynamic display and space environment. It can provide cultural support to the models and enrich their cultural connotation and sense of feeling. Appealing performance will provide a more profound understanding of the meaning of the fashion to the audiences.

\section{References}

[1] MENG Zhaolan. General Psychology[M].Beijing: Peking University Press. 2005:77-78.

[2] GUAN Jie. The analysis of model professional sense $[\mathrm{J}]$. Beauty and Age, 2004(5)55-56.

[3] Same as [2]

[4] CHEN Si. Art of Clothing Performance and Stage Culture[D]. Tianjin: Tianjin Polytechnic University, 2007:42. 
[5] ZHANG Yuan, WANG Jian. The discussion about atmosphere design of clothing performance[J]. Beauty and Age, 2003(12):64-65. 